\title{
Developing green photochemical approaches towards the synthesis of carbon nanofiber- and graphene-supported silver nanoparticles and their use in the catalytic reduction of 4-nitrophenol
}

\author{
María J. Fernández-Merino, Laura Guardia*, Juan I. Paredes, Silvia Villar-Rodil, \\ Amelia Martínez-Alonso, Juan M. D. Tascón \\ Instituto Nacional del Carbón, INCAR-CSIC, Apartado 73, 33080 Oviedo, Spain
}

\begin{abstract}
A green, photochemical approach for the liquid-phase synthesis of carbon nanomaterialsupported silver nanoparticles is proposed. The method is based on irradiating a colloidal dispersion containing the carbon nanomaterial, a metal precursor and an environmentally friendly reducing agent (bioreductant) with UV light at room temperature. Two representative carbon materials have been used, namely, platelet-type graphite nanofibers and graphene oxide. The experimental conditions and possible mechanisms that afford the photochemical growth of the nanoparticles on each carbon support are also investigated and discussed. In addition, the resulting carbon-silver nanoparticle hybrids are demonstrated to be notably effective catalysts for the reduction of 4-nitrophenol to 4-aminophenol with $\mathrm{NaBH}_{4}$. Particularly, the graphene oxide-based samples were seen to exhibit exceptional catalytic activity towards such reaction. Finally, it is also shown that with a proper choice of bioreductant the present UV approach can afford highly reduced graphene oxide samples comparable to those attained with well-known, efficient chemical reductants (e. g., hydrazine at $\sim 100{ }^{\circ} \mathrm{C}$ ), thus constituting an attractive room temperature alternative to such reduction methods.
\end{abstract}

\section{Introduction}

Due to their unique and tunable properties (optical, electronic, catalytic, etc.), noble metal nanoparticles (NPs) have been for the past two decades and continue to be the subject of research for their use in a range of applications that cover such diverse fields as optics, electronics, sensing, catalysis or biomedicine. ${ }^{1,2}$ Among noble metals, silver is particularly attractive with a view to many of these prospective uses owing to, e.g., its very high electrical conductivity, antibacterial activity and relatively low cost. From a catalysis perspective, silver nanoparticles (Ag NPs) are relevant to a number of important processes in the chemical industry, such as the selective oxidation of alkanes and alkenes, ${ }^{2}$ the hydrogenation of dyes $^{3}$ or the reduction of 4-nitrophenol (4-NP) to 4aminophenol (4-AP) as an intermediate step in the synthesis of pharmaceutical compounds (e.g., paracetamol). ${ }^{4}$ For their use in catalysis, the NPs are usually immobilized onto suitable substrates, such as silica, alumina, zeolites and different types of carbon materials, in order to (i) prevent their agglomeration and hence the reduction of their catalytic activity, and (ii) in some cases to enhance their efficiency through a proper interaction with the substrate. ${ }^{2}$

The synthesis of Ag NPs (whether supported or unsupported), and of noble metal NPs in general, is frequently carried out by means of wet chemical approaches, mainly because these methods are simple, versatile and scalable. ${ }^{5}$ Originally such methods tended to make extensive use of hazardous reagents and/or solvents, but increasing

\footnotetext{
* Corresponding author: Telephone number: (+34) 9851190 90. Fax number: (+34) 9852976 62. E-mail address: lauraguardia@incar.csic.es (L.Guardia)
} 
environmental awareness has driven in recent years a move towards the search of more sustainable preparation procedures. In particular, significant progress has been achieved on the green synthesis of unsupported Ag NPs using non- or low-toxic solvents, reductants and capping agents, and to a lesser extent more efficient heating systems. ${ }^{6-8}$ However, similar approaches aiming specifically at the preparation of substratesupported Ag NPs have been comparatively much less explored. In fact, in the case of carbon substrates only a limited number of reports, published very recently, have addressed the development of environmentally friendly production methods, ${ }^{9-14}$ and given the utility of these metal-carbon support systems in such relevant fields as, e.g., catalysis, ${ }^{15}$ further advances along this direction are clearly required.

Here, we report further developments of green methods for the liquid-phase synthesis of carbon-supported Ag NPs. Two different carbon materials relevant as catalyst supports have been chosen as substrates for this study: (i) platelet-type graphite nanofibers (PGNFs), a kind of carbon material with a surface made up exclusively of graphitic edge planes ${ }^{16}$ that has been postulated as a suitable support for metal catalysts, 15 and (ii) graphene derived from graphite oxide [reduced graphene oxide (RGO)], which is being very actively investigated at present as a support of metal and other nanostructures towards a number of applications. ${ }^{17,18}$ The method proposed here is of photochemical nature, making use of ultraviolet (UV) light to trigger the required reactions at room temperature, and as will be shown below, can be adapted to the specific characteristics of the carbon material and the solvent medium where the synthesis is carried out. In the case of graphene, this method also affords the simultaneous reduction of the precursor graphene oxide (GO) to a limited extent. Furthermore, the prepared PGNF- and RGO-Ag NP hybrids are shown to exhibit a remarkable catalytic activity in the reduction of 4-NP with $\mathrm{NaBH}_{4}$, thus illustrating their application potential.

On the other hand, using glutathione as reducing agent in the absence of the silver salt, GO is reduced to a large extent, similar to that attained heating at $95^{\circ} \mathrm{C}$ with the most efficient chemical reductants known till present, i. e., vitamin $\mathrm{C}$ and the widely used but toxic hydrazine. Such method can therefore be put forward as a convenient and particularly efficient way to reduce GO dispersions at room temperature in a relatively short time.

\section{Experimental section}

The PGNFs were acquired from Sigma-Aldrich and used as received. Aqueous dispersions of GO sheets were produced by exfoliation of graphite oxide as described previously. ${ }^{19}$ RGO was obtained by photochemically induced reduction of such dispersions. The preparation of the RGO- and PGNF-Ag NP hybrids was based on the irradiation with UV light at room temperature of a water/ethanol solution containing GO/PGNFs, $\mathrm{AgNO}_{3}$ as a metal precursor, glucose/pyridoxamine (PM) as an environmentally friendly reducing agent, ammonia, and in some cases polyvinylpyrrolidone (PVP) as a NP capping agent. The optimal concentrations for the preparation of the PGNF-Ag NP hybrid in ethanolic solution were $40 \mu \mathrm{M} \mathrm{AgNO}_{3}, 48$ $\mu \mathrm{M}$ PM, $0.002 \mathrm{wt} / \mathrm{vol} \mathrm{PVP}$, and $0.136 \mathrm{mg} \mathrm{mL}^{-1}$ PGNF. In the case of the RGO-Ag NP hybrid in aqueous solution, $0.5 \mathrm{mM} \mathrm{AgNO}_{3}, 2 \mathrm{mM}$ glucose, and $0.1 \mathrm{mg} \mathrm{mL}^{-1} \mathrm{GO}$. For the reduction of an aqueous dispersion of $\mathrm{GO}$ with glutathione in absence of silver salt, $2.5 \mathrm{mM}$ glutathione and $0.1 \mathrm{mg} \mathrm{mL}^{-1} \mathrm{GO}$. The $\mathrm{pH}$ was adjusted to 10 with ammonia. 10 $\mathrm{mL}$ of the resulting solutions were then irradiated with a UV spot lamp (BlueWave 50 unit, from Dymax) for a given time while bubbling them with an argon flow (45 $\mathrm{mL}$ 
$\left.\min ^{-1}\right)$. The UV light was emitted from the tip of a lightguide $5 \mathrm{~mm}$ in diameter that was positioned $\sim 15 \mathrm{~mm}$ above the surface of the solution, delivering an intensity of 1.0-1.2 $\mathrm{W} \mathrm{cm}{ }^{-2}$ in the $280-450 \mathrm{~nm}$ wavelength range as measured with a hand-held sensor (model UVM-CP, from UV-Consulting Peschl).

The catalytic activity of the carbon-supported Ag NPs was evaluated by investigating the reduction of 4-NP to 4-AP with $\mathrm{NaBH}_{4}$ at room temperature. The experiments were typically carried out by first diluting the aqueous/ethanolic suspension of RGO-Ag NP/PGNF-Ag NP hybrid, so as to obtain a concentration of Ag NPs in the resulting solution of $\sim 2-5 \times 10^{10} \mathrm{~mL}^{-1}$. Then, $25 \mu \mathrm{l}$ of a mixture of $5 \times 10^{-3} \mathrm{M}$ 4-NP and $3 \mathrm{M} \mathrm{NaBH}_{4}$ in water were added to $2.08 \mathrm{~mL}$ of the hybrid solution. The subsequent reduction of 4-NP was followed by means of UV-vis absorption spectroscopy. In order to assess the possibility of catalyst leaching, the "filtrate transfer" test $^{20}$ was carried out. For this test, first the catalyzed reaction is performed under the aforementioned conditions. After reaction completion, the carbon-supported Ag NPs are removed by centrifugation $(30 \mathrm{~min}, 20000 \mathrm{~g}$ ). Finally, the supernatant liquid is recovered and the 4-NP reduction is carried out in this medium keeping the other parameters unchanged.

Characterization of the hybrids was carried out by UV-vis absorption spectroscopy, field-emission scanning electron microscopy (FE-SEM), energydispersive X-ray (EDX) spectroscopy, atomic force microscopy (AFM), transmission electron microscopy (TEM), inductively coupled plasma mass spectrometry (ICP-MS) and X-ray photoelectron spectroscopy (XPS). UV-vis absorption spectra were recorded with a double-beam He $\lambda$ ios $\alpha$ spectrophotometer, from Thermo Spectronic. FE-SEM images and EDX spectra were taken on a Quanta FEG 650 apparatus (FEI Company) on specimens prepared by drop-casting a small volume of the dispersion of the hybrid onto a pre-heated $\left(\sim 50-60{ }^{\circ} \mathrm{C}\right)$ highly oriented pyrolytic graphite (HOPG) substrate and allowing it to dry under ambient conditions. A similar procedure was followed for preparing specimens for AFM, which was carried out under ambient conditions with a Nanoscope IIIa Multimode (Veeco) apparatus in the tapping mode of operation, using silicon cantilevers. TEM imaging was accomplished either with a JEOL 2011 operated at $200 \mathrm{kV}$ or with a JEOL $2000 \mathrm{EX}-\mathrm{II}$ instrument operated at $160 \mathrm{kV}$. To this end, the suspension of the hybrid was drop-cast onto a copper grid (200 mesh) covered with either a lacey or a continuous amorphous carbon film and allowed to dry. An ICP-MS 7500 ce (Agilent Technologies) instrument was employed for the ICP-MS measurements, using $\mathrm{He}$ as collision/reaction gas in the cell to avoid spectral interferences. For the determination of silver on the supported materials an aliquot of sample $(0.5 \mathrm{~mL})$ was centrifuged for $30 \mathrm{~min}$ at $20000 \mathrm{~g}$. After centrifugation the solid part was separated and treated with $0.5 \mathrm{~mL} \mathrm{HNO}_{3}$. The mixture was left for one hour and then diluted 5 times with ultrapure water, 1000 times with $\mathrm{HNO}_{3} 1 \%$ and $\mathrm{Rh}$ was added as internal standard in a final concentration of $5 \mathrm{ppb}$. The quantification of silver was done with external calibration, with standards prepared in $\mathrm{HNO}_{3} 1 \%$, and internal standard correction. XPS measurements were carried out on a SPECS system, working at a pressure of $10^{-7} \mathrm{~Pa}$ with a monochromatic $\mathrm{Al} \mathrm{K} \mathrm{K}_{\alpha} \mathrm{X}$-ray source $(1486.3 \mathrm{eV}, 100 \mathrm{~W})$. Specimens for XPS were prepared by casting the dispersion of the hybrids dropwise onto a metallic sample holder preheated at $\sim 50-60{ }^{\circ} \mathrm{C}$ until a dark film was seen to uniformly cover the sample holder.

\section{Results and discussion}

Several issues were taken into account in the implementation of our method, including the preferential use of non-toxic and environmentally benign solvents, 
reductants and capping agents, as well as the deployment of efficient energy sources to trigger the synthesis reaction (as opposed to the use of, e.g., conventional heating). ${ }^{6,8,21}$ Concerning the solvent medium, water is obviously the most environmentally friendly of all solvents, and should thus be the natural choice for the preparation of the hybrids. However, PGNFs are not colloidally stable in water, forming agglomerates than hinder the uniform decoration of the individual nanofibers with Ag NPs. In this case, ethanol was selected as an alternative solvent medium because it displays relatively low toxicity and environmental impact ${ }^{22}$ and was very recently shown to afford stable dispersions of individual PGNFs in significant concentrations (Fig. 1a-c). ${ }^{16}$ Moreover, being a versatile, low boiling point solvent, the use of ethanol can facilitate the subsequent processing of the prepared PGNF-Ag NP hybrids. On the other hand, it is known that both GO and RGO can form stable aqueous dispersions consisting of individual, singlelayer sheets (Fig, 1d-f). ${ }^{19}$ Therefore, the RGO-Ag NP hybrids were prepared in water. UV light was chosen as the energy source to promote the desired reaction, i.e. reduction of $\mathrm{Ag}^{+}$ions to yield metallic silver, and in the case of GO, also its conversion to RGO. In contrast to conventional or even microwave-assisted heating, with UV light the energy input to the process can in principle be directly targeted to the reacting molecules/species, thus avoiding heating up the bulk of the solution and allowing for the reaction to be carried out at ambient temperature and pressure. ${ }^{21}$ This is a particularly attractive feature when using low boiling point solvents and applies in the present case, since neither ethanol not water absorb UV photons from the wavelength spectrum emitted by the UV lamp used here.

Focusing first on the PGNFs in ethanol, we found that PM could trigger the reduction of the $\mathrm{Ag}^{+}$ions to yield metallic silver in the presence of UV light. PM is one of the naturally occurring forms of vitamin $\mathrm{B}_{6}$ and is a completely innocuous, environmentally friendly biomolecule. In the biochemical context, vitamin $\mathrm{B}_{6}$ has been reported to possess antioxidant activity, ${ }^{23}$ and therefore it is plausible that it can act as a reducing agent in a number of other, non-biological chemical reactions. However, to the best of our knowledge, its use and role in the photochemical synthesis of metal NPs has not been documented. FE-SEM revealed that UV irradiation of a PGNF dispersion in ethanol containing $40 \mu \mathrm{M} \mathrm{AgNO} 3$ as a metal precursor and $48 \mu \mathrm{M}$ PM (both soluble in ethanol) for $15 \mathrm{~min}$ led to the decoration of the nanofibers with NPs $\sim 30-80 \mathrm{~nm}$ in size (Fig. 2a). Control experiments indicated that NPs did not form when the solution was not irradiated with the UV lamp. In the absence of PM, however, a very small but finite number of NPs were generated. Ethanol could have acted itself as reducing agent, and indeed it has been reported to reduce $\mathrm{Ag}^{+}$ions to metallic silver NPs at sufficiently high temperatures. ${ }^{24}$ In the present case, ethanol was not directly heated by the UV lamp, but there was indication that the PGNFs were indeed warmed up, since the temperature of their dispersions in ethanol (without $\mathrm{AgNO}_{3}, \mathrm{PM}$ or any other reagents) rose by several degrees upon UV irradiation (from $\sim 20$ to $30^{\circ} \mathrm{C}$ ). PGNFs strongly absorb photons from the wavelength range emitted by the UV lamp used here ${ }^{16}$ and the subsequent relaxation of photoexcited electrons can lead to local heating of the nanofibers and, by thermal energy transfer, of the ethanol molecules around them, which in turn could trigger reduction of the $\mathrm{Ag}^{+}$ions to some extent. Nevertheless, this process was highly inefficient in producing significant numbers of NPs and most of the PGNFs did not become decorated with any. Only the use of PM afforded NPs in considerable quantities. As PM contains an aromatic ring, it strongly absorbs photons from the near and middle ranges of the UV spectrum. Thus, it can be hypothesized that the photo-excited PM $\left(\mathrm{PM}^{*}\right)$ can trigger the reduction of $\mathrm{Ag}^{+}$ions to metallic $\mathrm{Ag}$ NPs. This question will be further discussed below. 
In an attempt to control the size and increase the density of the generated NPs, the amounts of $\mathrm{AgNO}_{3}$ and $\mathrm{PM}$ in the medium as well as the $\mathrm{UV}$ irradiation time were changed. A general trend towards larger NP sizes with increasing amounts of $\mathrm{AgNO}_{3}$ and PM as well as irradiation time was observed, whereas NPs did not form when using lower concentrations of reagents. On the other hand, the use of PVP as a capping agent afforded NPs of smaller dimensions, mostly between 10 and $20 \mathrm{~nm}$. Furthermore, it was found that the process could be optimized, particularly in terms of attaining high NP densities, with a combination of PVP and ammonia in the ethanolic solution. The role of ammonia in the reported synthesis deserves some comment: $\mathrm{Ag}^{+}$ions possess a strong affinity towards ammonia molecules, forming the stable ionic complex $\left[\mathrm{Ag}\left(\mathrm{NH}_{3}\right)_{2}\right]^{+}$ (Tollens reagent). ${ }^{6}$ In aqueous medium, this complex has been shown to be a convenient precursor of silver nanostructures via reduction with either aldehydes or, more frequently, saccharides. ${ }^{6}$ In the present case, it can be argued that the $\left[\mathrm{Ag}\left(\mathrm{NH}_{3}\right)_{2}\right]^{+}$ complex is formed following addition of ammonia to the ethanolic solution of $\mathrm{AgNO}_{3}$. Reduction of the complex would be subsequently triggered by $\mathrm{PM}^{*}$, yielding small silver nuclei that would be stabilized by the ammonia ligands. Such stabilization would favor the formation of larger numbers of silver nuclei, which in turn would lead to the generation of a high density of NPs of smaller size (compared to the case where ammonia is not used) upon further growth of the nuclei, as was actually observed. Using the optimized amounts of reagents as specified in the Experimental section led to PGNFs decorated with large numbers of relatively small NPs (Fig. 2b). As could be expected, EDX spectroscopy indicated that carbon and silver were the dominant elements in the PGNF-NP hybrids (Fig. 2b, inset). The metallic character of the Ag NPs was deduced from the observation of a feature at about $410 \mathrm{~nm}$ in the UV-vis absorption spectrum of the hybrids (Fig. 2c), which can be attributed to the surface plasmon resonance (SPR) band typical of metallic silver nanostructures. ${ }^{25}$ Furthermore, XPS measurements revealed two well-defined and symmetrical peaks centered at 368.2 and $374.2 \mathrm{eV}$ (Fig. 2d), which can be ascribed to photoelectrons ejected from the $3 \mathrm{~d}_{5 / 2}$ and $3 \mathrm{~d}_{3 / 2}$ levels, respectively, of $\mathrm{Ag}(0) .{ }^{26}$ TEM imaging disclosed a highly uniform decoration of Ag NPs onto the PGNFs (Fig.2e). Detailed TEM images (e.g., Fig. 2f) were also employed to estimate NP sizes: a size distribution histogram obtained from the measurement of $~ 1000$ NPs is given as an inset to Fig. 2f, yielding an average size of $16 \pm 5 \mathrm{~nm}$. Virtually all the generated Ag NPs were anchored on the nanofiber surface and no stand-alone NPs were noticed, which can be put down to a strong interaction between metal clusters and the surface oxygen-containing groups present at edges and other defect sites of graphitic structures, including these nanofibers. ${ }^{15,16,27}$ The Ag concentration onto the PGNF determined by ICP-MS analysis showed a value of 13 ppm. Thus, with an average size of about $16 \mathrm{~nm}$ for the NPs, the Ag NP concentration on the PGNF was calculated to be $\sim 4 \times 10^{11} \mathrm{~mL}^{-1}$.

We now turn our attention to the preparation of RGO-Ag NP hybrids. Unlike the case of the PGNFs in ethanol, UV irradiation of an aqueous dispersion of GO sheets in the presence of the appropriate reagents including PM failed to yield Ag NPs in any significant amounts. Because GO possesses plenty of oxygen-containing functional groups and other structural defects where metal nuclei can anchor and subsequently grow, ${ }^{28,29}$ we believe that such result is not due to the use of this carbon substrate but rather to the solvent medium, which apparently plays a critical role in the performance of PM to trigger the reduction of $\mathrm{Ag}^{+}$ions. This question will be further discussed below. After testing several alternative biomolecules, glucose was found to be a good substitute for PM to grow Ag NPs on the GO sheets in aqueous medium. RGO-Ag NP hybrids prepared at moderate temperatures by simultaneous reduction of GO and silver 
ions with glucose in presence of PVP to guarantee the colloidal stability of GO have been very recently reported. ${ }^{30}$ However, in our case, the optimized procedure did not require the addition of PVP either to guarantee GO colloidal stability (as in the RGOAg NP hybrids previously reported in the literature $)^{30}$ or to control the NP size (as in the PGNF-Ag NP hybrids described above). Only $\mathrm{AgNO}_{3}$, glucose and ammonia were added to the aqueous GO dispersion, which was then irradiated for $15 \mathrm{~min}$. The results of a general characterization of the optimized hybrids are presented in Fig. 3. AFM imaging (Fig. 3a) revealed that the individual, single-layer GO sheets, identified as twodimensional, $\sim 1 \mathrm{~nm}$ thick objects in the images (see line profile in Fig. 3a), became uniformly decorated with a large number of bright dots. Such dots were about $20 \mathrm{~nm}$ high and were not present on the starting GO sheets (Fig. 1b), indicating that they correspond to the generated Ag NPs. Again, the observation of a SPR band at $402 \mathrm{~nm}$ in the UV-vis spectrum (Fig. 3c) and of two peaks at 368.3 and $373.2 \mathrm{eV}$ in the XPS spectrum (Fig. 3d) provides clear indication of the metallic character of the Ag NPs grown on the GO sheets. We also note that the SPR band of the GO-based hybrid (Fig. $3 \mathrm{c}$ ) is much more intense than that of its PGNF counterpart (Fig. 2c) if these bands are compared with the corresponding peak located in the $255-270 \mathrm{~nm}$ region. The latter is ascribed to $\pi \rightarrow \pi^{*}$ transitions in $\mathrm{C}=\mathrm{C}$ of graphitic carbon materials, ${ }^{16}$ and therefore can be associated to the PGNF and GO/RGO components of the hybrids. Such result suggests that a larger amount of Ag NPs has been generated per unit mass of carbon support when using GO compared with the PGNFs. In addition to solvent (water vs. ethanol) and reagent (glucose vs. PM) effects, this result could be due to the fact that GO possesses a much higher specific surface area than that of PGNFs, and consequently much more abundant surface sites for the anchoring of NPs per unit mass of the carbon support. Either polygonal or approximately rounded NPs were seen to decorate the GO sheets by TEM (Fig. 3b). Their size distribution (inset to Fig. 3b) ranges from 14 to 26 $\mathrm{nm}$, yielding average size of $19 \pm 4 \mathrm{~nm}$. The Ag concentration on the GO sheets was determined to be $52 \mathrm{ppm}$ by ICP-MS analysis. Using these values, the Ag NP concentration in the aqueous dispersion of the hybrids yielded $\sim 1 \times 10^{12} \mathrm{~mL}^{-1}$.

Apart from affording significant quantities of Ag NPs on the GO sheets, the method proposed here employing UV light and glucose was found to reduce GO itself. In fact, glucose has been previously reported to reduce $\mathrm{GO}$ although through heat treatment at $95{ }^{\circ} \mathrm{C} .{ }^{31}$ Indeed, from Fig. $3 \mathrm{c}$, there is a certain red-shift of the $\pi \rightarrow \pi{ }^{*}$ transition band associated to GO in the UV-vis absorption spectrum of the hybrid material from its initial value of $231 \mathrm{~nm}$. The magnitude of such red-shift is known to be a good indicator of the extent of GO reduction. ${ }^{32}$ To investigate in more detail the efficiency of the present approach towards reducing GO, its aqueous dispersion was irradiated with the UV lamp following the same procedure employed for the preparation of the hybrids with the exception that $\mathrm{AgNO}_{3}$ was not included in the solution. Fig. 4a (green squares) shows that the position of the absorption band associated to GO becomes increasingly red-shifted with irradiation time, reaching a plateau value $(262 \mathrm{~nm})$ after $60 \mathrm{~min}$. This value is only slightly higher than the one obtained by just irradiating GO, in the absence of glucose $\mathrm{e}^{33}$ and still far from that attained by means of chemical reduction at $95{ }^{\circ} \mathrm{C}$ using such powerful reducing agents as hydrazine or vitamin $\mathrm{C}$ at $95^{\circ} \mathrm{C}(268-270 \mathrm{~nm}),{ }^{32}$ which means that GO can be reduced only to a limited extent by UV irradiation in presence of glucose. In principle, this may come from an intrinsic limitation of the photochemical method itself or of the particular reductant used. To investigate this issue, glutathione (GSH), another previously reported bioreductant ${ }^{34}$ which does not absorb UV light (see below for the relevance of this point) was assayed. As shown in Fig. 4a (blue squares), UV irradiation of a dispersion of GO in the presence of GSH (2.5 mM, 5 
hours) led to a red-shift to $268-270 \mathrm{~nm}$. This demonstrates that the photochemical reduction method can be highly efficient, provided that a proper choice of bioreductant is made. Further confirmation of the successful reduction of GO by GSH was obtained by means of XPS high resolution $\mathrm{C}$ 1s core level spectra (Fig. 4b). Unreduced GO exhibits a strong component at $\sim 286.5 \mathrm{eV}$ (orange plot), implying that a large fraction of its carbon atoms are in an oxidized state; ${ }^{32}$ by contrast, treatment with UV light and GSH (blue plot) led to a marked decrease of this component, the main peak being located at $284.6 \mathrm{eV}$, which is assigned to carbon atoms in a graphitic, unoxidized environment. ${ }^{32}$ Reduction with glucose (Fig. 4b, green plot), being less effective, yields a similar $\mathrm{C} 1 \mathrm{~s}$ spectrum, but with a higher relative abundance of oxygen-containing groups. Both the reduction of GO using GSH as a reducing agent (at $50^{\circ} \mathrm{C}$ ) ${ }^{34}$ or by UV irradiation alone (photoinduced thermal reduction) ${ }^{33}$ have been recently reported, and they are less efficient than their combination described here, in terms of the extent of reduction achieved and/or the time required to its completion. Since the oxygencontaining functional groups in GO can only be thermally reduced at temperatures above $\sim 150{ }^{\circ} \mathrm{C}^{33}$, the local temperature at the UV photoirradiated sheets can be estimated to be at least $150{ }^{\circ} \mathrm{C}$. The synergistic effect between UV light and GSH could come from the fact that such relatively high temperature could kinetically assist reduction reactions between GO and GSH that are not favored at lower temperatures.

We now briefly discuss the possible mechanisms that allow the reduction of $\mathrm{Ag}^{+}$ ions to metallic Ag NPs using UV light and PM in ethanol but not in water, and why glucose can be a good substitute for PM in the latter medium. UV photon absorption should lead to a photoexcited molecule $\left(\mathrm{PM}^{*}\right)$, which could relax through a variety of processes. We hypothesize that one relaxation process involves the charge-transfer between $\mathrm{PM}^{*}$ and the solvent molecules. The fact that Ag NPs could be produced in ethanol but not in aqueous medium is consistent with the existence of solvent-specific interactions. Indeed, solvent-specific charge-transfer between a photoexcited aromatic molecule and solvent molecule has been previously reported in the literature. ${ }^{35}$ As ethanol is known to be an efficient hole scavenger, its reaction with $\mathrm{PM}^{*}$ would be expected to yield a reduced PM molecule, which in turn could take part in the formation of silver nanoparticles. Another possibility for the interaction between a photoexcited aromatic molecule and ethanol with a subsequent reduction of $\mathrm{Ag}^{+}$has just been reported by Yang et al. ${ }^{36}$ This involves the formation of a biradical between the molecules, which in turn would be able to transfer electrons to $\mathrm{Ag}^{+}$ions, thereby reducing them.

On the other hand, hole scavenging of $\mathrm{PM}^{*}$ by water molecules can be expected to generate hydroxyl radicals, which possess a strongly oxidizing capability and are wellknown to easily degrade many organic compounds. ${ }^{37}$ Consequently, we surmise that in aqueous medium both PM and its reduced counterpart will be readily decomposed in the presence of UV light and thus will not be able to reduce $\mathrm{Ag}^{+}$ions to metallic Ag NPs. Evidence in support of this hypothesis was provided by the UV-vis absorption spectra of PM solutions in either water or ethanol recorded before and after irradiation with the UV lamp (Fig. 5). In aqueous medium, fresh PM solutions exhibit three characteristic absorption bands at 220, 254 and $328 \mathrm{~nm}$ (Fig. 5a, blue plot), which disappeared after UV irradiation of the solution for $15 \mathrm{~min}$ (Fig. 5a, black plot) pointing to the decomposition of the molecule. On the other hand, the three distinctive bands of PM (Fig. 5b, red plot), were preserved to a certain extent after UV irradiation for $15 \mathrm{~min}$ in ethanol (Fig. 5b, black plot), which confirms that PM endures the irradiation process at least for a period of $15 \mathrm{~min}$ in such solvent without total degradation. 
In principle, the use of other molecules that can be photoexcited with UV light should also afford the growth of Ag NPs in ethanol. Indeed, experiments carried out with pyrogallol, which is another reducing agent with strong absorbance in the near to middle UV range, indicated that NPs could also be produced, albeit of a somewhat larger size. On the other hand, when an UV light-transparent reductant was employed instead (for instance, $\mathrm{NaBH}_{4}$ ), NPs were hardly generated, similar to what has been described above for the control experiments in the absence of PM. In the same line of reasoning, UV light-absorbing molecules should be generally inappropriate for the synthesis of Ag NPs in aqueous medium. In this case, glucose was found to be a good substitute for PM to induce the growth of Ag NPs on GO and also to afford a certain degree of reduction of GO itself. Because glucose is transparent to UV light, the mechanisms that allow these processes to take place must be completely different to those occurring with PM in ethanol. We believe that the GO sheets play a central role in these processes. Evidence in this regard was obtained from the observation that Ag NPs did not form when GO was excluded from the synthesis solution. By contrast, the growth of Ag NPs in ethanol using PM should not require the presence of a carbon substrate, and indeed we were able to produce Ag NPs in this medium in the absence of the PGNFs. As is well known, GO also exhibits strong absorbance in the 280-450 nm wavelength. ${ }^{33}$ However, unlike the case of PM, reaction with water molecules and the subsequent generation of highly reactive hydroxyl radicals are not thought to be dominant processes involving photoexcited GO. If this was the case, we would expect further oxidation and possibly disintegration of the GO sheets by the hydroxyl radicals, ${ }^{38}$ but rather the opposite trend (i.e., deoxygenation) has been observed in UVirradiated GO, ${ }^{33}$ likely as a result of its local heating. Thus, in the presence of a suitable reducing agent such as glucose, reduction of $\mathrm{GO}$ and of $\mathrm{Ag}^{+}$ions should become kinetically favored by the locally high temperatures, leading to the decoration of the sheets with metallic Ag NPs.

Finally, to demonstrate the suitability of these PGNF- and RGO-supported Ag NPs as effective catalysts, we investigated their performance in the reduction of 4-NP to 4AP with $\mathrm{NaBH}_{4}$ in aqueous medium as a model reaction. ${ }^{39}$ The reaction progress can be readily followed with UV-vis absorption spectroscopy, by monitoring the drop in intensity of the absorption peak of the deprotonated form of 4-NP generated upon addition of $\mathrm{NaBH}_{4}$ (Fig. 6a, dark yellow plot, band at $400 \mathrm{~nm}$ ) as a function of reaction time. The rates of reduction were independent of the concentration of $\mathrm{NaBH}_{4}$ since this reagent was used in large excess (1:600). Therefore, the reduction reaction can be expected to obey a pseudo-first-order kinetic behavior with respect to 4-NP, so that

$$
\frac{d[4-\mathrm{NP}]}{d \mathrm{t}}=-k_{\text {app }}[4-\mathrm{NP}]
$$

where [4-NP] is the concentration of 4-nitrophenoxide and $k_{a p p}$ is the observed rate constant. Indeed, the evolution of absorbance at $400 \mathrm{~nm}$ with time for the reaction catalyzed with PGNF- and RGO-supported Ag NPs, (Fig. 6a, green and black squares, respectively) could be well fit to an exponential decay (Fig. 6b, solid lines) yielding rate constants $k_{a p p}=(4.2 \pm 0.9) \times 10^{-3} \mathrm{~s}^{-1}$ and $(9 \pm 2) \times 10^{-2} \mathrm{~s}^{-1}$, respectively. We stress that no conversion of 4-NP to 4-AP was observed to occur either in the absence of the hybrids or when only the nanofibers or the graphene sheets (without NPs) were present in the reaction solution, confirming the catalytic role played by the NPs. We also note that essentially full conversion of 4-NP to 4-AP was achieved in the experiments, because the band at $400 \mathrm{~nm}$ virtually came to disappear. The very same weak absorption band noticed after reaction completion at about $396 \mathrm{~nm}$ (Fig. 6a, violet plot) was already observed in the reaction solution in the absence of any added 4-NP, and can be ascribed 
to the SPR band of the Ag NPs. This band is known to blue-shift (in the present case, from 410 to $396 \mathrm{~nm}$ ) as a result of the injection of electrons from the nucleophilic $\mathrm{BH}_{4}{ }^{-}$ anion onto the Ag NP surface. ${ }^{39}$ Likewise, the background absorbance observed both before and after reaction completion in Fig. 6a can be attributed to the carbon material. In order to verify that the catalytic process is heteregeneous, i. e., that the reaction takes place exclusively or at least predominantly on the surface of the supported nanoparticles, the possibility of leaching was studied. We employed the filtrate transfer test, ${ }^{20}$ which consisted of performing the 4-nitrophenol reduction using the supernatant obtained by centrifugation of the sample after carrying out a first reaction cycle. It was observed that the 4-nitrophenol reaction reaction either did not occur in such supernatant (with the GO support) or it was dramatically slowed down (with the PGNFs support). Consequently, both catalysts can be considered to be essentially heterogeneous with minimal leaching.

To compare the catalytic performance of the carbon-supported Ag NPs with that of other catalysts reported in the literature, the observed rate constant was normalized to either the surface area or the mass of silver employed per unit volume, yielding estimated values of $0.1 \mathrm{~L} \mathrm{~s}^{-1} \mathrm{~m}^{-2}$ and $3.8 \mathrm{~L} \mathrm{~s}^{-1} \mathrm{~g}^{-1}$ for the PGNF-Ag NP system and $6 \mathrm{~L} \mathrm{~s}$ ${ }^{1} \mathrm{~m}^{-2}$ and $150 \mathrm{~L} \mathrm{~s}^{-1} \mathrm{~g}^{-1}$ for the RGO-Ag NP system, respectively. Table 1 gathers normalized rate constants obtained in recent years with efficient catalysts based on $\mathrm{Ag}$ NPs. ${ }^{40-52}$ As can be seen, the catalyst prepared here using GO as a support exhibits an outstanding catalytic activity, even better than the most remarkable values reported until present. This feature combined with its long-term colloidal stability (several months at least) make it stand out as an excellent catalyst for this reaction.

\section{Conclusions}

A green photochemical approach based on the use of intense UV light and appropriate environmentally friendly biomolecules has been shown to be efficient towards the room-temperature, liquid-phase synthesis of silver nanoparticles supported onto two different carbon nanomaterials, namely, platelet-type graphite nanofibers and graphene oxide. The resulting nanostructured hybrids exhibited long-term colloidal stability in water or ethanol, which can be advantageous with a view to their further manipulation and use in practical applications. Thus, it was demonstrated that the hybrids possess a high catalytic activity in the reduction of 4-nitrophenol to 4-aminophenol with $\mathrm{NaBH}_{4}$. In particular, the catalytic activity of the graphene oxide-based hybrids was found to be one of the highest that have ever been reported with silver nanoparticle-based catalysts for such a reaction. Finally, a synergistic effect was found between UV light and glutathione (a well-known natural antioxidant) towards the reduction of graphene oxide sheets, affording a degree of deoxygenation in a room-temperature process that is only matched by the use of the most efficient chemical reductants of graphene oxide (e.g., hydrazine) at higher $\left(80-100^{\circ} \mathrm{C}\right)$ temperatures.

\section{Acknowledgements}

Financial support from the Spanish MINECO and the European Regional Development Fund (project MAT2011-26399) is gratefully acknowledged. M.J.F.-M. is thankful for the receipt of a pre-doctoral contract (FPI) from MINECO.

\section{References}

1 P. K. Jain, X. Huang, I. H. El-Sayed and M. A. El-Sayed, Acc. Chem. Res., 2008, 41, 1578. 
2 J. M. Campelo, D. Luna, R. Luque, J. M. Marinas and A. A. Romero, ChemSusChem, 2009, 2, 18.

3 Z. -J. Jiang, C. -Y. Liu and L. -W. Sun, J. Phys. Chem. B, 2005, 109, 1730.

4 N. Pradhan, A. Pal and T. Pal, Colloid Surf. A-Physicochem. Eng. Asp., 2002, 196, 247.

5 C. Burda, X. Chen, R. Narayanan and M. A. El-Sayed, Chem. Rev., 2005, 105, 1025.

6 V. K. Sharma, R. A. Yngard and Y. Lin, Adv. Colloid Interface Sci., 2009, 145, 83.

7 S. Iravani, Green Chem., 2011, 13, 2638.

8 M. N. Nadagouda, T. F. Speth and R. S. Varma, Acc. Chem. Res., 2011, 44, 469.

9 S. S. Kurva, J. Kathi, K. Y. Rhee and S. J. Park, J. Nanosci. Nanotech., 2011, 11, 4463.

10 W. Yuan, Y. Gua and L. Li, Appl. Surf. Sci., 2012, 261, 753.

11 J. Fan, Z. Shi, Y. Ge, J. Wang, Y. Wang and J. Yin, J. Mater. Chem., 2012, 22, 13764.

12 L. Chen, H. Xie and W. Yu, J. Mater. Sci., 2012, 47, 5590.

13 Y. Zhang, S. Liu, L. Wang, X. Qin, J. Tian, W. Lu, G. Chang and X. Sun, RSC Adv., 2012, 2, 538.

14 X. Wang, P. Huang, L. Feng, M. He, S. Guo, G. Shen and D. Cui, RSC Adv., 2012, 2, 3816.

15 P. Serp, J. L. Figueiredo (eds.), in Carbon Materials for Catalysis, John Wiley \& Sons, Hoboken, 2009.

16 L. Guardia, J. I. Paredes, S. Villar-Rodil, J. -N. Rouzaud, A. Martínez-Alonso and J. M. D. Tascón, Carbon, 2013, 53, 222.

17 X. Huang, X. Qi, F. Boey and H. Zhang, Chem. Soc. Rev., 2012, 41, 666.

18 S. Bai and X. Shen, RSC Adv., 2012, 2, 64.

19 J. I. Paredes, S. Villar-Rodil, P. Solís-Fernández, A. Martínez-Alonso and J. M. D. Tascón, Langmuir, 2009, 25, 5957.

20 H. Cong and J. A. Porco Jr., ACS Catal., 2012, 2, 65.

21 M. Lancaster, in Green Chemistry: An Introductory Text, Royal Society of Chemistry, Cambridge, 2002.

22 C. Capello, U. Fischer and K. Hungerbühler, Green Chem., 2007, 9, 927.

23 P. Stocker, J. -F. Lesgards, N. Vidal, F. Chalier and M. Prost, Biochim. Biophys. Acta, 2003, 1621, 1.

24 A. Pal, S. Shah and S. Devi, Mater. Chem. Phys., 2009, 114, 530.

25 Y. Sun and Y. Xia, Analyst, 2003, 128, 686.

26 B. V. Crist, in Handbook of Monochromatic XPS Spectra. The Elements \& Native Oxides. XPS International LLC. California, USA, 2004, vol. 1, pp.1-4.

27 A. Felten, C. Bittencourt, J. -F. Colomer, G. Van Tendeloo and J. -J. Pireaux, Carbon, 2007, 45, 110.

28 I. Fampiou and A. Ramasubramaniam, J. Phys. Chem. C, 2012, 116, 6543.

29 M. N. Groves, C. Malardier-Jugroot and M. Jugroot, J. Phys. Chem. C, 2012, 116, 10548.

30 X. -Z. Tang, X. Li, Z. Cao, J. Yang, H. Wang, X. Pu and Z. -Z. Yu. Carbon, 2013, DOI: 10.1016/j.carbon.2013.02.058.

31 C. Zhu, S. Guo, Y. Fang and S. Dong, ACS Nano, 2010, 4, 2429.

32 M. J. Fernández-Merino, L. Guardia, J. I. Paredes, S. Villar-Rodil, P. SolísFernández, A. Martínez-Alonso and J. M. D. Tascón, J. Phys. Chem. C, 2010, 114, 6426. 
33 L. Guardia, S. Villar-Rodil, J. I. Paredes, R. Rozada, A. Martínez-Alonso and J. M. D. Tascón, Carbon, 2012, 50, 1014.

34 T. A. Pham, J. S. Kim, J. S. Kim and Y. T. Jeong, Colloid. Surf. A, 2011, 384, 543.

35 J. Choi, W. Choi and B. J. Mhin, Environ Sci Technol., 2004, 38, 2082.

36 Y. Yang, L. Liu, H. Yin, D. Xu, G. Liu, X. Song and J. Liu, J. Phys. Chem C, 2013, 117, 11858.

37 M. B. Ray, J. P. Chen, L. K. Wang and S. O. Pehkonen, in Advanced Physicochemical Treatment Processes, Handbook of Environmental Engineering, eds. L. K. Wang, Y. -T. Hung and N. K. Shammas, 2006, vol. 4, pp 463-481.

38 P. Solís-Fernández, J. I. Paredes, S. Villar-Rodil, L. Guardia, M. J. FernándezMerino, G. Dobrik, L. P. Biró, A. Martínez-Alonso and J. M. D. Tascón, J. Phys. Chem. $C, 2011,115,7956$.

39 N. Pradhan, A. Pal and T. Pal, Colloid Surf. A-Physicochem. Eng. Asp., 2002, 196, 247.

40 K. O. Santos, W. C. Elias, A. M. Signori, F. C. Giacomelli, H. Yang and J. B. Domingos, J. Phys. Chem. C, 2012, 116, 4594.

41 R. Eising, A. M. Signori, S. Fort and J. B. Domingos, Langmuir, 2011, 27, 11860.

42 A. M. Signori, K. O. Santos, R. Eising, B. L. Albuquerque, F. C. Giacomelli and J. B. Domingos, Langmuir, 2010, 26, 17772.

43 J. T. Zhang, G. Wei, T. F. Keller, H. Gallagher, C. Stötzel, F. A. Müller, M. Gottschaldt, U. S. Schubert and K. D. Jandt, Macromol. Mater. Eng., 2010, 295, 1049.

44 S. Harish, R. Sabarinathan, J. Joseph and K. L. N. Phani, Mater. Chem. Phys., 2011, 127, 203.

45 A. Murugadoss and A. Chattopadhyay, Nanotechnology, 2008, 19, 015603.

46 P. Zhang, C. Shao, Z. Zhang, M. Zhang, J. Mu, Z. Guo and Y. Liu, Nanoscale, 2011, 3, 3357.

47 Y. Lu, Y. Mei, M. Schrinner, M. Ballauff, M. W. Möller and J. Breu, J. Phys. Chem. $C, 2007,111,7676$.

48 Y. Lu, Y. Mei, M. Ballauff and M. Drechsler, J. Phys. Chem. B, 2006, 110, 3930.

49 B. Baruah, G. J. Gabriel, M. J. Akbashev and M. E. Booher, Langmuir, 2013, 29, 4225.

50 Md. H. Rashid and T. K. Mandal, J. Phys. Chem. C, 2007, 111, 16750.

51 W. Zhang, F. Tan, W. Wang, X. Qiu, X. Qiao and J. Chen, J. Hazard. Mater., 2012, 217-218, 36.

52 S. Tang, S. Vongehr and X. Meng, J. Mater. Chem., 2010, 20, 5436. 
Table 1. Comparison of areal rate constants obtained for the reduction of $p$-nitrophenol with $\mathrm{NaBH}_{4}$ using a number of Ag NP-based catalysts.

\begin{tabular}{|c|c|c|c|}
\hline \multirow[t]{3}{*}{ Catalytic system } & \multirow{3}{*}{$\begin{array}{l}\text { Areal rate } \\
\left(\mathrm{L} \mathrm{m}^{-2} \mathrm{~s}^{-1}\right)\end{array}$} & Rate normalized & \multirow[t]{3}{*}{ Reference } \\
\hline & & to $\mathrm{Ag} \quad \mathrm{NP}$ & \\
\hline & & $\begin{array}{l}\text { concentration } \\
\left(\mathrm{L} \mathrm{g}^{-1} \mathrm{~s}^{-1}\right)\end{array}$ & \\
\hline PGNF-supported Ag NP hybrids & 0.1 & 3.8 & $\begin{array}{l}\text { Present } \\
\text { work }\end{array}$ \\
\hline RGO-supported Ag NPs hybrids & 6 & 150 & $\begin{array}{l}\text { Present } \\
\text { work }\end{array}$ \\
\hline $\begin{array}{l}\text { Ag NP-linear polyethylene imine } \\
\text { colloidal systems }\end{array}$ & 1.7 & & 40 \\
\hline $\begin{array}{l}\text { Colloidal, dextran T500-stabilized Ag } \\
\text { NPs }\end{array}$ & 1.4 & & 41 \\
\hline $\begin{array}{l}\mathrm{Ag} \text { NP-polyethyleneimine derivative } \\
\text { composite }\end{array}$ & 0.57 & & 42 \\
\hline Ag NPs embebbed in polymeric microgel & $0.12-0.20$ & & 43 \\
\hline $\begin{array}{l}\text { Colloidal, aminosilicate-stabilized Ag } \\
\text { NPs }\end{array}$ & $0.08-0.20$ & & 44 \\
\hline Chitosan-Ag NP composite & 0.15 & & 45 \\
\hline $\begin{array}{l}\mathrm{Ag} \text { NPs on polymer-derived carbon } \\
\text { nanofibers }\end{array}$ & 0.15 & 3.3 & 46 \\
\hline Ag NPs in polyelectrolyte brush particles & 0.08 & & 47 \\
\hline Ag NPs in core-shell polymeric microgel & 0.05 & & 48 \\
\hline $\begin{array}{l}\mathrm{Ag} \text { NPs stabilized by cationic } \\
\text { polynorbornenes }\end{array}$ & & 1.4 & 49 \\
\hline Spherical (and dendritic) Ag NPs & $0.02(*)$ & $1.4-4.0$ & 50 \\
\hline Ag nanodendrites & 0.02 & 0.01 & 51 \\
\hline $\begin{array}{l}\text { Ag NPs embedded in porous carbon } \\
\text { spheres }\end{array}$ & & 0.01 & 52 \\
\hline
\end{tabular}

(*) The areal rates could only be estimated for the spherical Ag NPs with the available data. 
Figure 1.
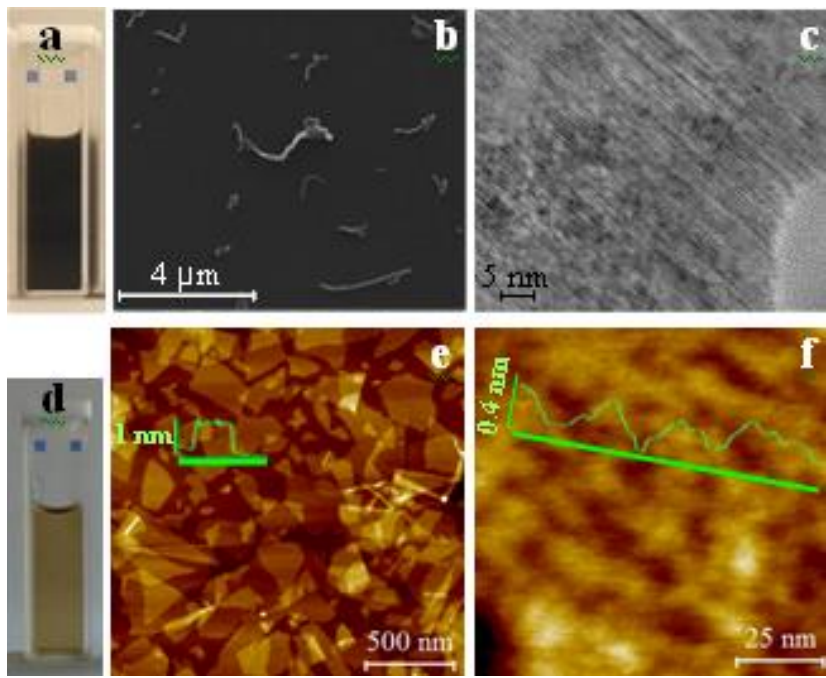


\section{Figure 2.}
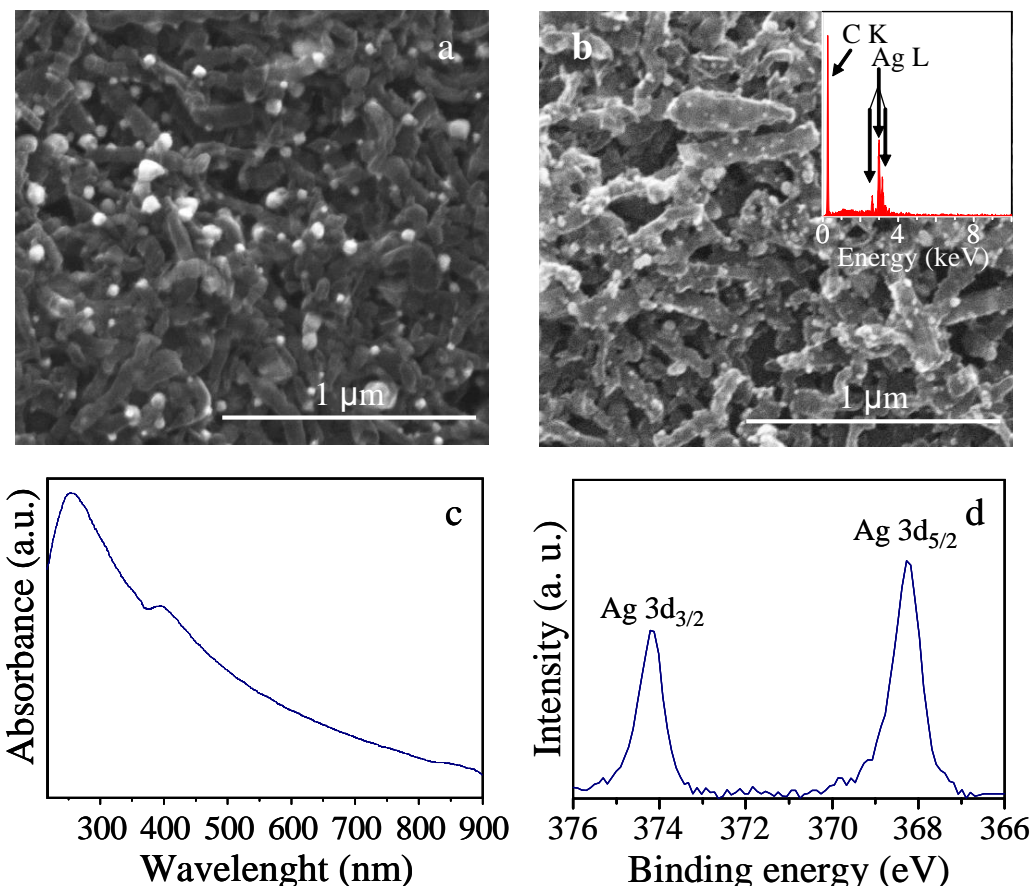

Wavelenght (nm)
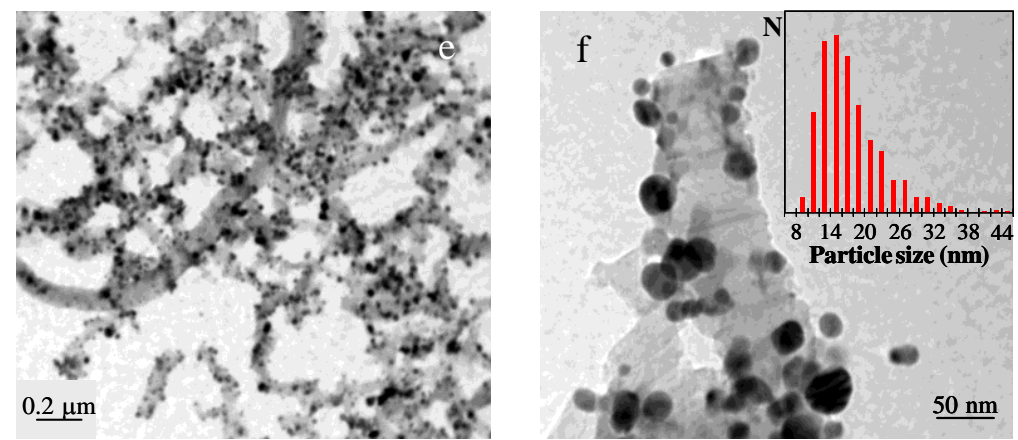
Figure 3.
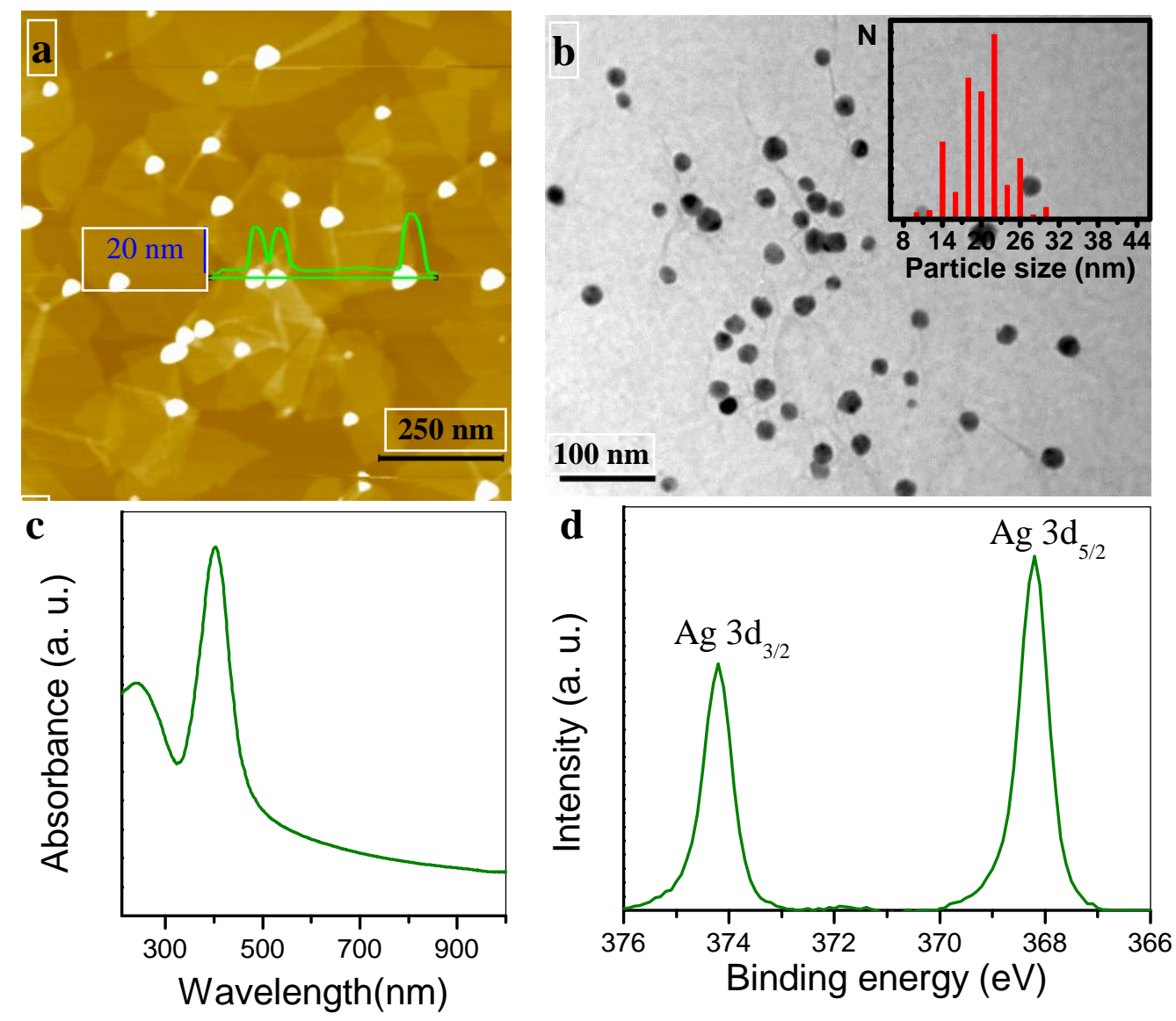
Figure 4.
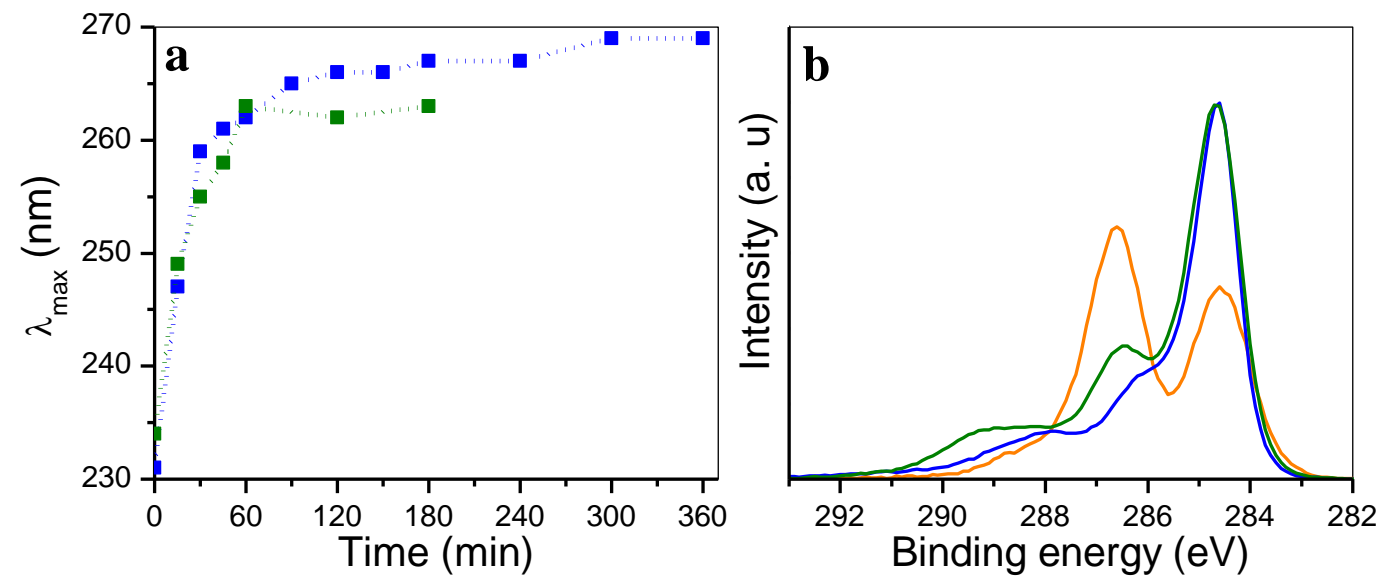
Figure 5.
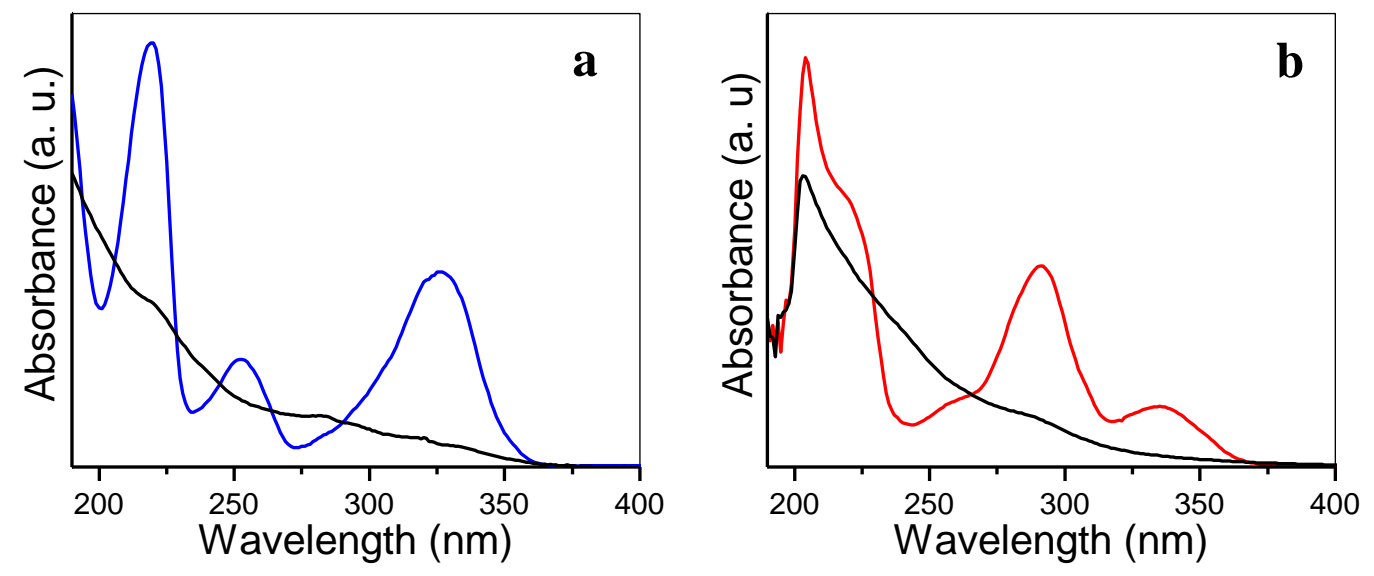
Figure 6.

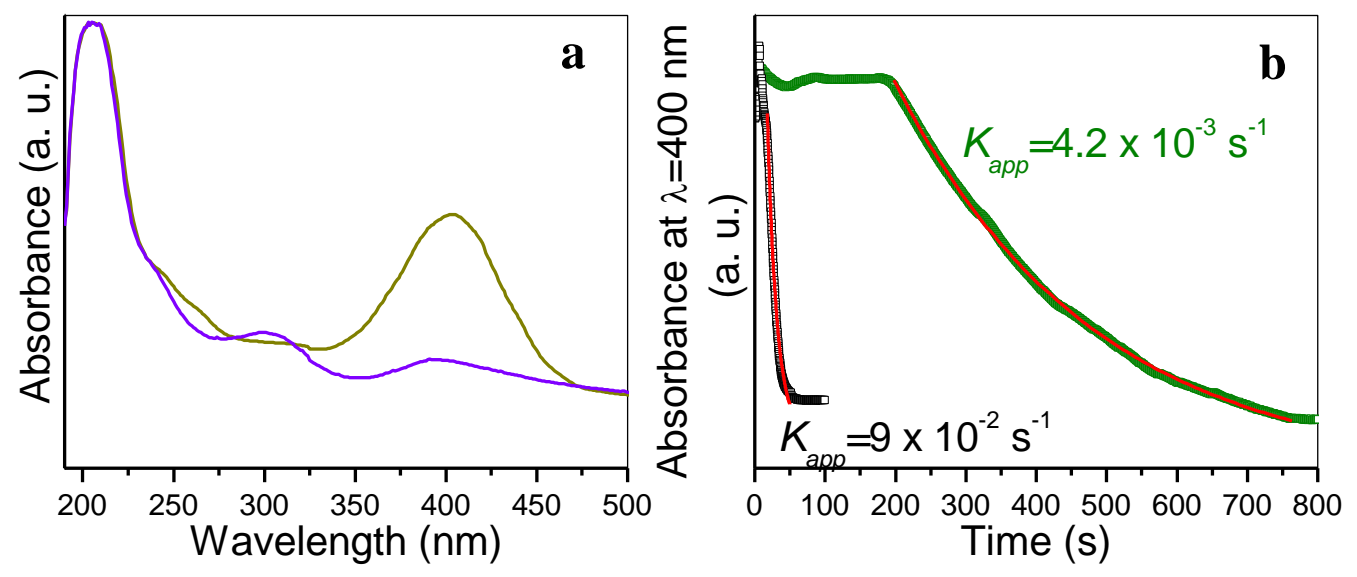




\section{Captions to figures.}

Figure 1. (a) Digital photograph of a PGNF dispersion in ethanol. (b) FE-SEM image of the PGNF dispersion drop-cast onto HOPG. (c) HRTEM image of a PGNF, where its platelet morphology is clearly seen. (d) Digital photograph of an aqueous GO dispersion. (e, f) AFM images of (e) the GO dispersion drop-cast onto HOPG, and (f) an individual sheet on the same atomically flat substrate. In each case a height profile taken along the green line is shown superimposed on the image. (e) The lateral size of the sheets is in the submicron range, while their apparent thickness (measured as their height relative to the substrate) is $\sim 1 \mathrm{~nm}$, which is consistent with the sheets being single-layer objects. (f) Given that the sheets are deposited on an atomically flat substrate, the observed extremely small roughness $(<0.4 \mathrm{~nm})$ must be an intrinsic feature of their structure.

Figure 2. (a,b) FE-SEM images of silver nanoparticles grown onto PGNFs using $\mathrm{AgNO}_{3}$ and PM in the absence (a) and presence (b) of PVP and ammonia. EDX spectrum is given as an inset to (b). (c,d) UV-vis absorption spectrum in ethanol and Ag $3 \mathrm{~d}$ XPS of the sample shown in (b). (e, f) TEM images at different magnifications of silver nanoparticles grown onto PGNFs using $\mathrm{AgNO}_{3}$ and $\mathrm{PM}$ in the presence of PVP and ammonia. A histogram of nanoparticle size distribution is given as an inset to (f).

Figure 3. (a) AFM and (b) TEM images of GO-Ag NP hybrids. A histogram of nanoparticle size distribution is given as an inset to (b). (c) UV-vis absorption and (d) Ag 3d XPS spectrum of the sample shown in $(a, b)$.

Figure 4. (a) Wavelength of the absorption maxima for the $\pi \rightarrow \pi^{*}$ transition of unsaturated carbon bonds of graphene oxide irradiated with UV light for different periods of time in the presence of glucose (green squares), and of GSH (blue squares). (b) C1s core level XPS spectra of the starting graphene oxide (orange plot), and that irradiated with UV light treated in the presence of glucose (green plot), and of GSH (blue plot).

Figure 5. UV-vis absorption of aqueous (a) and ethanolic (b) PM solutions before (colored line) and after (black line) UV irradiation for $15 \mathrm{~min}$.

Figure 6. (a) UV-vis absorption spectra for the reduction of 4-NP to 4-AP with $\mathrm{NaBH}_{4}$ at room temperature in the presence of PGNF-supported silver nanoparticles just before (dark yellow plot) and after (violet plot) reduction. (b) Plot of the time evolution of the intensity of the peak at $400 \mathrm{~nm}$ of 4-nitrophenolate ion in presence of the PGNF-Ag NP (green squares) or RGO-Ag NP (black squares) hybrids. The experimental kinetic profiles could be well fitted to exponential decay functions (residuals in the $\pm 0.03 \%$ ), which are shown as overlaid orange and red lines, respectively. Experimental conditions: $[4-\mathrm{NP}]=0.06 \mathrm{mM} ;\left[\mathrm{NaBH}_{4}\right]=35.6 \mathrm{mM}$; $[\mathrm{Ag} \mathrm{NP}] \sim 1-1.5 \mu \mathrm{g} \mathrm{mL}^{-1}$. 